DOI: $10.21767 / 2471-299 X .1000016$

\title{
Parenting Care Model is a Rehabilition for Learning Disabled Child
}

\author{
Saffiah Ani Binti Ismaill ${ }^{1}$, Puspa Liza Binti Ghazali ${ }^{2}$, Mustafa Bin Mamat ${ }^{3}$ and Zabidin bin Salleh ${ }^{4}$ \\ ${ }^{1}$ Faculty of Economic and Management Sciences, Gong Badak Campus, 21300 Kuala Terengganu, Malaysia \\ ${ }^{2}$ Faculty of Medical, Universiti Sultan Zainal Abidin, Kota Campus, Jalan Sultan 20400 Kuala Terengganu, Malaysia \\ ${ }^{3}$ Faculty of Informatics and Computing, Universiti Sultan Zainal Abidin, TembilaCampus, 22200 Besut, Terengganu, Malaysia \\ ${ }^{4}$ School of Informatics and Applied Mathematics, Universiti Malaysia Terengganu 21300 Kuala Terengganu, Malaysia
}

*Corresponding author: Saffiah Ani BI, Faculty of Economic and Management Sciences, Gong Badak Campus, 21300 Kuala Terengganu, Malaysia, Tel: +60145045465; E-mail: saffiah91@gmail.com

Rec date: Aug 23, 2015; Acc date: Feb 19, 2016; Pub date: Feb 23, 2016

Copyright: (C) 2016 Ismail SAB, et al. This is an open-access article distributed under the terms of the Creative Commons Attribution License, which permits unrestricted use, distribution, and reproduction in any medium, provided the original author and source are credited.

\section{Abstract}

Each day, millions of children with learning disabilities face tasks that, for reasons unknown to them, never become automatic and always seem to stand in the way of their progress as students. For parents and teachers of a struggling child, the picture is not much clearer or any less frustrating. Rehabilition is beginning at home for the parents to detect their kids whether in learning disability category. Learning Disability kids need 3 things to survive in the real world by using parenting care due to parenting skill, learning skill and nutrition. Parenting skill is the way how the parents can hand the learning disability kids to manage their daily life. Learning skill is the knowledge on how to teach the kids to learn mathematics (calculation skill) and language (read, comprehension skill and writing skill). Lastly, learning disability kids also need the best nutrition for their brain system, neuro system, blood system and digestion system. Therefore, parenting care is the first aid for learning disability kids for survival on the reality with their normal kids` friends.

Keywords: Rehabilition; Learning disability kid; Parenting care; Parenting skill; Learning skill; Nutrition

\section{Introduction}

Back to 1800 , there was a research on soldiers and their damaged brain due to war and mental impairment by Franz Joseph Gall [1]. Study on brain-related was started to emerge and brought to the learning disabilities term as the formal education imposed [2]. Studies on learning disabilities are still emerging day by day resulted with varieties of definitions and explanations on learning disabilities. Before society is aware about this issue, people or kids with learning disabilities are mistakenly called as stupid, lazy and under achiever due to their reaction on daily life activities are different when compared with peer. Late growth [3], difficulty in reading [4] and problem with body systems are among the symptoms shown by kids with learning disabilities. With the advantages of lots of researches, it had helped millions of parents to recognize similar symptoms of learning disabilities so that they can take a fast action such as referring their kids to child psychiatrics for further action.

Year by year, as statistically proved, the number of registered people with learning disabilities in Malaysia are increasing from 134, 659 during 2011 to 165, 281 during 2012 [5]. Learning disabled people in fact are brighter than peer. However, with the difficulties they are facing will affect their daily life starting from school year till old days. Lots of technique and incentives had been done by governments and privates organizations in helping these people to undergo a normal life. Understanding the root of learning disabilities problem, the symptoms and other related things not only help learning disabled people but also can help normal people to be with them and give a non-stop support.

Human abilities such as talking and eating are depending solely with central nervous system. However, due to several conditions such as during pregnancy; alcohol or drug use during pregnancy; child birth complication and terminal brain damage due to accident are recognized by researchers as the causes of learning disabilities. Mostly all the factors for learning disabilities are insulting the central nervous system and damaging certain part of brain such as the frontal lobe for reading and focusing [6], parietal lobe for mathematical reasoning [7] and temporal lobe for memorizing [6]. Even though there are parts of brain damaged, this kind of people are gifted such as Albert Einstein and Leonardo Da Vinci. From the factors found from lots of researches, learning disabilities are defined by Sharfi and Rosenblum [8] as follows:

"A term for a large group of neurological disorders caused by deficits in the central nervous system which influence the individual's ability to efficiently maintain process or conveys information to others."

As briefly stated in the previous paragraph, the causes of learning disabilities can be divided into three phases; before birth, during child birth and after birth [9]. What is happening during these three phases and how are they interfering with kids' central nervous system. 


\section{Before birth}

What is taken by the pregnant mothers play an important role during this stage? Even for non-pregnant mothers, healthy nutrition is a key for healthy life, both for mother and fetus. During the development of fetus till birth, any things consumed by the mother will be absorbed by the fetus trough the umbilical cord. The central nervous system of fetus will be interfered if the mother consumes drug or alcohol during pregnancy [10] and cigarette smoking [11]. The pregnant mother emotion must always stabilized and in good and positive mood. Depression, stress and anxiety will affect the infant development [12]. Fetus development also interferes if mother does not get enough nutrition [13].

A study conducted on the autoimmune disease during pregnancy on learning disabled kids. After testing 58 children whose mothers are with systematic lupus erythematous (SLE), this study found that sons of women with SLE are significantly to have learning disabilities compared with daughters of women with SLE. At the end of the port, researchers conclude that the fetal brain of mail offspring is attacked by the maternal antibodies which known as anti-Ro/La [14].

Other than the immune system, chromosomal conditions also can be credited for learning disabilities. There are lots of evidence that relate genetic and autism. However, there is no specific initial diagnostic evaluation of patients with autism. Hence, a clinical genetic testing had been conducted for autism spectrum disorder (ASD) where the testing were using G-banded karyotype, fragile $X$ testing and chromosomal microarray (CMA) to test for submicroscopic genomic deletions and duplications. Finally, the researchers conclude that CMA should be suggested as the initial evaluation for autism due to its highest detection rate among clinically available genetic test [15].

\section{During birth}

Complication during the delivery process has a great impact to baby especially to his or her tiny brain. In Scotland, most of kids which are placed under special educational need (SEN) had less birth weight and this is associated with sensory, physical or motor impairments and intellectual impairments [16]. Interrupted oxygen supply to the baby for a significant length of time can insult the baby's brain. The disruptions and disorders of pregnancy, labor and delivery and the neonatal period are known as obstetric complications. Prenatal drug exposure, poor maternal nutrition, minor physical anomalies and birth complications are among the obstetric complications. Even though babies are safely born, yet they have to face with the long-term effects such as problematic behavior due to the brains development were affected during complications [17].

\section{After birth}

The strength of kids' body cannot be compared with adults' body. Their body still in development state and it can be supported by healthy nutrition and, clean and safe environment. However, not all kids get the same opportunity to live with a complete and good condition for their growth. Even today, we live with advance technology and science researches, malnutrition problems still cannot be solved. Poor environment conditional leads to learning disabilities. Child abuse and severe head injury which brings damage to the brain also included as the factors [9].

People with learning disabilities have average and above average IQ level. However, they have impairments with psychological process related to learning. These impairments also can be listed as the symptoms of learning disabilities and by knowing the symptoms, kids with learning disabilities can get early help before it is too late to do so. Learning disabled kids usually have problem in 1) understanding and expressing information using words, 2)organizing visual information, 3) carrying out hand-eye activities, 4) identifying and manipulating speech sounds, 5) speed of taking in and using out information, 6) holding information in mind while also using the information and 7) planning and organizing [18].

\section{Common type of learning disabilities}

Specific learning disabilities: Dyslexia is a normally heard by society due to it is the common learning disabilities among people since dyslexics are simply diagnosed by observing they ability in reading. People with dyslexia are known as dyslexic and they have a difficulty in using and processing of linguistics and alphabetic letters. This occurs due to the left hemisphere of brain is damaged and activities related with reading are interrupted [19]. Other learning disability is dysgraphia. It is known for problem with handwriting where the handwriting is difficult to read [20]. Writing is an essential component to learn by kids and having dysgraphia, without getting any earlier help will lead to problem during adulthood. Those kids with dysgraphia take a long time to write and they required more in-air time than typically developing writers [21]. Next is dyscalculia and it is known for disability in recognizing number and do simple arithmetical problem [22]. Dyslexia, dysgraphia and as stated before, dyscalculia are known as specific learning disabilities due to the disorders for one or more of the basic psychological processes such as reading, writing and mathematical calculation [23].

Attention deficit / hyperactive disorder (ADHD): Focusing during learning session is a basic condition for kids to absorb and restore any new information or knowledge learnt from parents at home or teachers at school. In reality, not all kids have this ability. They only can give their attention or focus just for a short time span. This condition is called attention deficit disorder or ADD. Kids with ADD are mistakenly recognized as lazy but how to prove it is not when they have tried 10 times harder than their peer [24]? Other disability that interrupts the learning process and kids which cannot stay calm and focus is known as attention deficit hyperactivity disorders or ADHD. Activities involved with focus such as following a continuous command or driving, are very intense for them [25].

Autism spectrum disorder (ASD): Autism spectrum disorder (ASD) or shortly know as autism is a complex neurobehavioral disorder that includes impairments in developmental language 
and communication deficits, social interaction and repetitive behaviors [15]. Autistic people usually have problem with their digestion system as it has abnormal gut flora. As the gut flora is important in human immunity system, any interference on the gut flora directly gives an effect to the immunity system. It is showed with the appearance of allergies, asthma and eczema [26].

The symptoms showed typically appear between 18-month and 6-year of age. Since this type of disorder is on socialization and communication, its only can be detected when babies are normally started to talk and socialize in their second year [27].

\section{Problem Statements}

Prophet Muhammad (PBUH) said, stomach is a home (source) of all illness and abstinence from gluttony is the best drug. When we are looking at kids with learning disabilities, most of them are suffering with allergies to anything in the environment. They also have problem with their digestive system, respiratory system (asthma) and frequent ear infections, to state some. All these are disturbing their learning processes and to socialize. As stated by Dr Natasha CampbellMcBride, she never met with her patients without mental conditions without digestion problem. This is included people with learning disabilities.

\section{Literature Review}

Consuming the right amount of nutrients is important for kids' growth. However, even they are consuming a healthy nutrition; bad digestive system cannot digest certain nutrient due to the gastrointestinal problem [28].

Autism is strongly linked with this condition. Gastrointestinal problem and autism are strongly related. Diarrhea, constipation, bloating and / or gastro esophageal reflux are among the problem faced by kids with autism. These problems contribute to frustration, decreased ability to concentrate and behavior problems [29]. What is happening to the digestive system and how digestive disorder has effect on brain?

Inside of human digestive system, there live bacteria, viruses, yeast and worm and of course, there is large number of them and they are known as gut flora. Their presence is not wasted as they are vital in creating a healthy gut system. This healthy gut only can be created if and only if the good bacteria are conquering our digestive system. They have a powerful control on bad bacteria, viruses and yeast. Hence, no bad things will happen to our gut. However, this condition can be reversal if good bacteria are die and finally, the bad side conquering out gut and do their work such as yeasts They will produce toxins in our gut such as alcohol, acetaldehyde, dermorphin and deltorphin. Any food intake will be digested by the abnormal mass of microbes and they will convert those foods into hundreds of toxins. As these toxins get through the damaged gut wall, which is due to abnormal gut flora, and transmitted through blood stream and reach the brain. Certain parts of brain will be damaged base on the type of these toxins attack to particular lipoproteins. As a result, different symptoms will appear depending on which part of the brain is clogged with these toxins.

Kids' brains are still the developing brains and it only halted or suspended by any external disturbance. When brain is damaged and no action is taken, this will lead to long-lasting or permanent consequences [30]. Referring back to the definition of learning disabilities together with the factors, sentence like central nervous system repetitively appear. Normally, learning disabled kids have problem with reading and writing. This is due to in one kid; he or she can have more than one learning disability such as ADHD and dyslexia in the same time. The symptoms appeared mostly similar even kids are diagnosed with different type of learning disabilities and the symptoms such as problem with reading, writing and basic mathematic, sleeping disorder, lack of focus and digestive disorder [1, 8]. Our central nervous system consists of brain and spinal cord. As a general knowledge, brain is the important organ in living things as it can be assumed as the head quarter which control and monitoring all activities inside of living things body. Healthy brain brings to good body coordination and if otherwise, life will be challenging. When discussing about people with learning disabilities, what it can be said is, their brains are working in different way, not as the normal brain. In other word, there are certain parts of their brains are damaged and the damaged parts will disturb basic life activities such as organizing things according to priority, receiving simultaneous command and communication skills but put in our mind, they are not stupid just because of their disabilities and most them have above average intelligent quotient (IQ) [31].

\section{Discussion}

This part will discuss on the suitable basic nutrient supplement to be suggested in helping kids with learning disabilities. Vitamin $C$ for gut healing and omega-3 fatty acid with DHA and EPA; vitamin D and methionine will be suggested as the nutrient supplement needed in treating both gut and brain.

\section{Vitamin C treatment for immunity system and digestive system}

Vitamin $C$ is among the vital nutrients needed by human body for various biochemical and physiological processes. Human body does not have an ability to synthesize its own vitamin $C$ due to the absence of enzyme, I-gulonolactone oxides in liver. Hence, we have to consume it through tablets, fruits and vegetables. This vitamin helps to regulate nervous system, needed for tissue growth and wound healing, and increase the absorption of nutrients in gut [32].

How vitamin $C$ works for better immunity system and digestive system especially for learning disabled people? Due to the leaky gut syndrome, due to the toxins released by the abnormal gut flora, the undigested food and toxins are easily entering into the bloodstream, circulating inside of human body and reach certain body system, especially central 
nervous system; and interfere it. The immune system activates due to the presence of foreign objects. Since vitamin $C$ is vital for the better function of immune system, it will prevent them from reaching other body organ such as brain [33]. Other than helping the immune system, vitamin $\mathrm{C}$ also helps in healing the leaky gut as this vitamin has the ability to stimulate the collagen synthesis, one of the components in healing process [34].

\section{Omega-3 fatty acid DHA and EPA treatment for damaged brain}

Based on literature review, learning disabled kids can be helped by supplying them continuously with suitable supplement. A study was conducted on 19 learning disabled kids with treatment of nutrient supplement such as ascorbic acid, manganese and zinc. This study found a positive change in their learning achievement and behavior [35]. Basically, learning disabled kids have problems in learning due to their brain and digestive system's condition. With suitable nutrient treatment, damaged brain and digestive disorder can be cured and covered to the normal stage.

Brain is created with special ability. Its can back to normal if treated with right nutrient and enough exercise. It is known as brain capability of plasticity [36]. A clinical study was conducted to test the ability of damaged brain in five groups of 16 adult male Sprague-Dawley rats back to normal by treating them with omega-3 fatty acid docohexanoic acid (DHA) and eicosapentaenoic acid (EPA). This study concluded that DHA and EPA help in increasing the serum levels and if given before the brain damage, it can reduce the injury impact. When the tested rats were treated with high doses of DHA over 30 days after the injury, they showed a positive response to anatomical injury across low to high doses of omega-3 fatty acid DHA and EPA [37].

Omega-3 long-chain polyunsaturated fatty acid (PUFA) including EPA and DHA are dietary fats with lots of health benefits. They play a vital role in many parts of body especially cell membranes and act as anti-inflammatory processes and in the viscosity of cell membranes [38]. Brain cells or neurons have a high concentration of DHA in the membrane phospholipids. Cooperation of DHA and phospholipids is important in improving the efficiency of synaptic membrane vesicles in fusion events which is fundamental for neurotransmission [39]. The DHA presence is important in the formation of new membrane for recovery of damaged brain [36].

In the nutshell, the frontal lobe for reading and focusing; parietal lobe for mathematical reasoning; temporal lobe for memorizing and other damaged brain parts can be healed and be recovered to its typical condition and functions.

\section{Vitamin $\mathrm{D}$ and methionine}

Learning disabled kids tend to lose their focus especially during a class. This is due to the frontal lobe; one of the brain parts is not functioning well. This part plays a vital role in memorizing and focusing [40]. By consuming a suitable amount of vitamin D3, cognitive disorder such as focusing can be recovered. A study conducted by researchers in Qatar with 1, 331 kids of ADHD and 1, 331 normal kids as the control. They found that kids with ADHD have the highest vitamin $D$ deficiency compared with the normal kids [41].

Other than lack in focus, learning disabled kids also tend to depress with their condition. Being called as stupid due to their disabilities and less moral support will get the factor of depression. If no action taken, they have a tendency for them to get the worst mental problem such as schizophrenia. Other than undergoing counseling; methionine, an anti-depressant, it is easy to get since it is available in term of pills or in commercial balm such as Vicks. The study had been conducted on S-adenosyl-I-methionine (SAMe) and the study got the positive result in reducing the depression. It is also safe and effective [42].

\section{Conclusion}

Our abilities to focus, write and other cognitive activities are strongly associated with our brain. God creates human brain with the ability to recover to its normal condition with the help of enough healthy nutrition. Human digestion system plays an important role in supporting our brain to function well. You are what you eat. Knowing exactly what are we consuming daily is very important since it is not only for our brain but also for our body system. There is lots of nutrition needed by them but we only choose omega-3 fatty acid, vitamin C and D, and methionine in this article. These nutrition is just for the basic recovery of damaged gut and brain of kids with learning disabilities. Starting from healing the leaky gut, it will give the positive consequences for the healing the damaged brain such as for the autistics, ADHD and dyslexic. Parenting care which involved three important skill such as parenting skill, nutrition care and learning skill will help the kids with learning disabilities to survive in the real life as the normal people in the future.

\section{Acknowledgement}

I would like to thank for RACE Grant, UNISZA of sponsoring this research and also to FRGS Grant, UMT of funding the article publication fees.

\section{References}

Hallahan DP, Mercer CD (2001) Learning Disabilities: Historical Perspectives. Executive Summary.

Linden M, Habib T, Radojevic V (1996) A controlled study of the effects of EEG biofeedback on cognition and behavior of children with attention deficit disorder and learning disabilities. Biofeedback Self Regul 21: 35-49.

3. National Early Childhood Intervention Council (2013) Children with Disabilities in Malaysia-Mapping the Policies, Programmes, Interventions and Stakeholders. 
4. Büttner G, Hasselhorn M (2011) Learning disabilities: Debates on definitions, causes, subtypes, and responses. International Journal of Disability, Development and Education 58: 75-87.

5. United Nation Children Fund Malaysia (2014) Children with disability in Malaysia.

6. MayField Brain, Spain (2013) Anatomy of the brain.

7. Butterworth B, Varma S, Laurillard D (2011) Dyscalculia: from brain to education. Science 332: 1049-1053.

8. Sharfi K, Rosenblum S (2015) Sensory modulation and sleep quality among adults with learning disabilities: a quasiexperimental case-control design study. PLoS One 10: e0115518.

9. Holland K (2011) Factsheet: Learning disabilities.

10. Pressinger RW (1997) Alcohol exposure during pregnancy: Links to learning disabilities, ADD and behavior disorders.

11. Pressinger RW (1998) Cigarette smoking during pregnancy: Links to learning disabilities attention deficit disorder-A.D.D. Hyperactivity and behavior disorders.

12. Dunkel Schetter C, Tanner L (2012) Anxiety, depression and stress in pregnancy: implications for mothers, children, research, and practice. Curr Opin Psychiatry 25: 141-148.

13. Keenan, K, Bartlett TQ, Nijland M, Rodriguez JS, Nathanielsz PW, et al. (2013) Poor nutrition during pregnancy and lactation negatively affects neurodevelopment of the offspring: evidence from a translational primate model. Am J Clin Nutr 98: 396-402.

14. Ross G, Sammaritano L, Nass R, Lockshin M (2003) Effects of mothers' autoimmune disease during pregnancy on learning disabilities and hand preference in their children. Arch Pediatr Adolesc Med 157: 397-402.

15. Shen Y, Dies KA, Holm IA, Bridgemohan C, Sobeih MM, et al. (2010) Clinical genetic testing for patients with autism spectrum disorders. Pediatrics 125: e727-735.

16. MacKay DF, Smith GC, Dobbie R, Pell JP (2010) Gestational age at delivery and special educational need: retrospective cohort study of 407,503 schoolchildren. PLoS Med 7: e1000289.

17. Ishikawa S, Raine A (2003) Obstetric complications and aggression. Encyclopedia on early childhood development 1-6.

18. Hargreaves $\mathrm{H}$, Rowbotham M, Phillips M (2009) A hand book on learning disabilities.

19. Scerri TS, Schulte-Körne G (2010) Genetics of developmental dyslexia. Eur Child Adolesc Psychiatry 19: 179-197.

20. Berret S (2009) Learning disabilities 101.

21. Engel-Yeger B, Nagauker-Yanuv L, Rosenblum S (2009) Handwriting performance, self-reports, and perceived selfefficacy among children with dysgraphia. Am J Occup Ther 63: 182-192.

22. Geary (2004) Mathematics and Learning Disabilities. J Learn Disabil 37: 4-15.

23. American Psychiatric Association (2013) Diagnostic and Statistical Manual of Mental Disorders $\left(\mathrm{DSM}-5^{\circ}\right)$. American Psychiatric Pub.

24. Smith SL (2002) What do parents of children with learning disabilities, ADHD, and related disorders deal with? Pediatr Nurs 28: 254-257.
25. Brown TE (2006) Attention deficit disorder: The unfocused mind in children and adults. Yale University Press.

26. Campbell-McBride N (2005) Gut and psychology syndrome (GAP syndrome or GAPS)

27. Rodriguez AM (2011) Autism spectrum disorders. Minneapolis: Twenty- First Century Books.

28. Collins SM, Bercik P (2009) The relationship between intestinal micro biota and the central nervous system in normal gastrointestinal function and disease. Gastroenterology 136: 2003-2014.

29. Adams JB, Johansen LJ, Powell LD, Quig D, Rubin RA (2011) Gastrointestinal flora and gastrointestinal status in children with autism--comparisons to typical children and correlation with autism severity. BMC Gastroenterol 11: 22.

30. Kalia M (2008) Brain development: anatomy, connectivity, adaptive plasticity, and toxicity. Metabolism 57 Suppl 2: S2-5.

31. Eaton H (2010) Brain School: Stories of Children with Learning Disabilities and Attention Disorders who Changed Their Lives by Improving Their Cognitive Functioning. Glia Press.

32. Iqbal K, Khan A, Khattak MMAK (2004) Biological significance of ascorbic acid (Vitamin C) in human health-a review. Pakistan J of Nutrition 3: 5-13.

33. Resnick C (2010) Nutritional protocol for the treatment of intestinal permeability defects and related conditions. Natural Medicine Journal 2: 14-23.

34. Naidu KA (2003) Vitamin $C$ in human health and disease is still a mystery? An overview. Nutr J 2: 7.

35. Carlton RM, Ente G, Blum L, Heyman N, Davis W, et al. (2000) Rational dosages of nutrients have a prolonged effect on learning disabilities. Altern Ther Health Med 6: 85-91.

36. Gomez-Pinilla F, Gomez AG (2011) The influence of dietary factors in central nervous system plasticity and injury recovery. PM R 3: S111-116.

37. Mills JD, Bailes JE, Sedney CL, Hutchins H, Sears B (2011) Omega-3 fatty acid supplementation and reduction of traumatic axonal injury in a rodent head injury model: Laboratory investigation. J of Neurosurgery 114: 77-84.

38. Swanson D, Block R, Mousa SA (2012) Omega-3 fatty acids EPA and DHA: health benefits throughout life. Adv Nutr 3: 1-7.

39. Valenzuela B (2009) Docosahexaenoic acid (DHA), an essential fatty acid for the proper functioning of neuronal cells: their role in mood disorders. Grasas y aceites 60: 203-212.

40. de Souza LC, Guimarães HC, Teixeira AL, Caramelli P, Levy R, et al. (2014) Frontal lobe neurology and the creative mind. Front Psychol 5: 761.

41. Kamal MM (2014) Is High Prevalence of Vitamin D Deficiency a Contributory Factor for Attention Deficit Hyperactivity Disorder in Children and Adolescents? In 2014 AAP National Conference and Exhibition. American Academy of Pediatrics.

42. Mischoulon D, Fava M (2002) Role of S-adenosyl-L-methionine in the treatment of depression: a review of the evidence. Am J Clin Nutr 76: 1158S-61S. 